MATHEMATICS OF COMPUTATION

Volume 75, Number 255, July 2006, Pages 1351-1366

S 0025-5718(06)01825-4

Article electronically published on February 27, 2006

\title{
LOW RANK UPDATE OF SINGULAR VALUES
}

\author{
DELIN CHU AND MOODY CHU
}

\begin{abstract}
The notion of a low rank update arises in many important applications. This paper deals with the inverse problem of updating a rectangular matrix by additive low rank matrices so as to reposition the associated singular values. The setting is analogous to the classical pole assignment problem where eigenvalues of a square matrix are relocated. Precise and easy-to-check necessary and sufficient conditions under which the problem is solvable are completely characterized, generalizing some traditional Weyl inequalities for singular values. The constructive proof makes it possible to compute such a solution numerically. A pseudo algorithm is outlined.
\end{abstract}

\section{INTRODUCTION}

A low rank update of a data matrix is a task of critical importance in many disciplines. The notable BFGS method, for example, is a classical rank-2 update scheme employed in practical optimization for the Jacobian matrix. The list of other applications includes image compression, noise reduction, seismic inversion, and so on. One aspect of update is to "passively" approximate a given set of data by low rank matrices. Principal component analysis, factor retrieval, latent semantic indexing, regularization for ill-posed problems, and so on are some typical areas where low rank approximation is desired. Practical means to tackle the low rank approximation include the truncated singular value decomposition method [14], the Lanczos bidiagonalization process [20] and the Monte-Carlo algorithm [17]. A framework of structured low rank approximation has been discussed in 8]. Another aspect of update is to "actively" control the behavior of the resulting system by specifying some of the parameters that effectuate the dynamics. The state feedback pole assignment problem, for example, is to find a matrix $F \in \mathbb{R}^{m \times n}$ such that the matrix $A+B F$, where $A \in \mathbb{R}^{n \times n}$ and $B \in \mathbb{R}^{n \times m}$ are given, has a prescribed set of eigenvalues. These eigenvalues are often referred to as the poles of the underlying system. Such an eigenvalue reassignment problem might arise from the scenario where the state $\mathbf{x}(t) \in \mathbb{R}^{n}$ of a certain physical system under the dynamic state equation

$$
\dot{\mathbf{x}}(t)=A \mathbf{x}(t)+B \mathbf{u}(t)
$$

Received by the editor December 17, 2004 and, in revised form, April 1, 2005.

2000 Mathematics Subject Classification. Primary 68F18, 93B55, 15A18.

Key words and phrases. Singular values, low rank update, interlacing properties, pole assignment.

This research was supported in part by the National Science Foundation under grants DMS0073056 and CCR-0204157. 
is to be controlled by the input $\mathbf{u}(t) \in \mathbb{R}^{m}$. One classical process in control theory is to select the input $\mathbf{u}(t)$ so that the dynamics of the resulting $\mathbf{x}(t)$ is driven into to a certain desired state. In the state feedback control, the input $\mathbf{u}(t)$ is selected as a linear function of current state $\mathbf{x}(t)$, i.e.,

$$
\mathbf{u}(t)=F \mathbf{x}(t) .
$$

In this way, the system (1.1) is changed to a closed-loop dynamical system:

$$
\dot{\mathbf{x}}(t)=(A+B F) \mathbf{x}(t) .
$$

A general goal in such a control scheme is to choose the gain matrix $F \in \mathbb{R}^{m \times n}$ so as to achieve stability and to speed up response. To accomplish this goal, the problem can be translated into choosing $F$ so as to reassign eigenvalues of the matrix $A+B F$.

The pole assignment problem has been thoroughly studied in the literature. See, for example, 3, 18, 22, 26]. Let $\lambda(M)$ denote the spectrum of a square matrix $M$. A standard result in this field is that, given any set of $n$ complex numbers $\left\{\lambda_{1}, \cdots, \lambda_{n}\right\}$ which is closed under complex conjugation, a matrix $F \in \mathbb{R}^{m \times n}$ exists such that $\lambda(A+B F)=\left\{\lambda_{1}, \cdots, \lambda_{n}\right\}$ if and only if

$$
\operatorname{rank}[A-\mu I, B]=n, \quad \text { for all } \mu \in \mathbb{C} .
$$

The condition (1.4) usually is referred to as the pair $(A, B)$ being controllable. In the single-input case where $m=1$, it is further known that the pole assignment problem, if solvable, has a unique solution. On the other hand, it can be proved that

$$
\bigcap_{F \in \mathbb{R}^{m \times n}} \lambda(A+B F)=\{\mu \in \lambda(A) \mid \operatorname{rank}[A-\mu I, B]<n\},
$$

implying that for a certain peculiar pair $(A, B)$ of matrices the eigenvalues of $A$ cannot be reassigned by any $F$. This kind of unassignable matrix pair forms a zero measure set.

Analogous to the task of reassigning eigenvalues of a square matrix, it is natural to ask whether the singular values of a rectangular matrix can be arbitrarily reassigned via additive low rank matrices. More specifically, the following special type of inverse singular value problem (ISVPrk) is studied in this paper. For convenience, we use $\sigma(N)$ to denote henceforth the set of singular values of a general matrix $N$.

(ISVPrk) Given a matrix $A \in \mathbb{R}^{m \times n}(m \geq n)$, an integer $n \geq$ $\ell>0$ and real numbers $\beta_{1} \geq \beta_{2} \geq \cdots \geq \beta_{n} \geq 0$, find a matrix $F \in \mathbb{R}^{m \times n}$ such that $\operatorname{rank}(F) \leq \ell$ and $\sigma(A+F)=\left\{\beta_{1}, \beta_{2}, \cdots, \beta_{n}\right\}$.

The state feedback pole assignment problem is but a special case of the much broader class of inverse eigenvalue problems which has attracted remarkable attention in recent years. See, for example, [2, 7] and the many references cited therein. In contrast, the inverse singular value problems have not received as many studies. Some earlier results that can be considered as inverse problems include the de Oliveira theorem [10] on the principal elements and singular values, the Weyl-Horn theorem [15, 25. on the relationship between singular values and eigenvalues, and the Sing-Thompson theorem [21, 24] on the majorization between the diagonal elements and singular values. Some related numerical work can be found in [4, 5, 6]. However, we are not aware of any discussion on the problem formulated as the ISVPrk. It might be worth noting that the ISVPrk can also be considered as a 
generalization of the additive inverse eigenvalue problem that has been well studied in the literature.

Although an inverse singular value problem can be recast as a specially structured inverse eigenvalue problem (see [7]), the existing theory does not provide us a clue on when the ISVPrk is solvable. Our main contribution in this paper is that we completely characterize the necessary and sufficient condition under which the above ISVPrk is solvable. Our theory not only generalizes the classical Weyl inequalities [16, 23] to multiple ranks, but also offers a constructive proof which can be implemented as a numerical means to find the solution.

\section{RANK ONE UPDATE}

We shall begin with the case where $F$ is of rank one only. The discussion will be used as a building block to extend to the general case.

Given any fixed column vector $\mathbf{b} \in \mathbb{R}^{m}$, consider first the case where $F$ is of the form $F=\mathbf{b f}^{\top}$ for some undetermined column vector $\mathbf{f} \in \mathbb{R}^{n}$. Choose the Householder transformation $Q_{\mathbf{b}} \in \mathbb{R}^{m \times m}$ so that

$$
Q_{\mathbf{b}}^{\top} \mathbf{b}=\left[\begin{array}{c}
b_{0} \\
\mathbf{0}
\end{array}\right]
$$

where $b_{0}=\|\mathbf{b}\|_{2} \in \mathbb{R}$ and $\mathbf{0}$ denotes throughout the paper a zero vector of appropriate size. Denote the product $Q_{\mathrm{b}}^{\top} A$ in blocks, i.e.,

$$
Q_{\mathbf{b}}^{\top} A=\left[\begin{array}{c}
\mathbf{a}_{\mathbf{b}}^{\top} \\
A_{\mathbf{b}}
\end{array}\right],
$$

with $\mathbf{a}_{\mathbf{b}} \in \mathbb{R}^{n}$ and $A_{\mathbf{b}} \in \mathbb{R}^{(m-1) \times n}$. Depending on whether $m=n$ or $m>n$, the singular value decomposition of $A_{\mathbf{b}}$ is of the form

$A_{\mathbf{b}}=U_{\mathbf{b}}\left[\begin{array}{ccccc}\gamma_{1} & & & & 0 \\ & \gamma_{2} & & & 0 \\ & & \ddots & & \vdots \\ & & & \gamma_{n-1} & 0\end{array}\right] V_{\mathbf{b}}^{\top} \quad$ or $\quad A_{\mathbf{b}}=U_{\mathbf{b}}\left[\begin{array}{cccc}\gamma_{1} & & & \\ & \gamma_{2} & & \\ & & \ddots & \\ & & & \gamma_{n} \\ \mathbf{0} & & & \mathbf{0}\end{array}\right] V_{\mathbf{b}}^{\top}$,

respectively. For any $\mathbf{f} \in \mathbb{R}^{n}$, denote

$$
\left(\mathbf{a}_{\mathbf{b}}^{\top}+b_{0} \mathbf{f}^{\top}\right) V_{\mathbf{b}}=\left[f_{1}, f_{2}, \cdots, f_{n}\right] .
$$

Define the matrix $A(\mathbf{f}) \in \mathbb{R}^{(n+1) \times n}$ as a function of $\mathbf{f}$ by

$$
A(\mathbf{f})=\left[\begin{array}{ccccc}
f_{1} & f_{2} & \cdots & f_{n-1} & f_{n} \\
\gamma_{1} & & & & \\
& \gamma_{2} & & & \\
& & \ddots & & \\
& & & \gamma_{n-1} & \\
& & & & \gamma_{n}
\end{array}\right]
$$

where $\gamma_{n}=0$ if $m=n$. Then, we know that

$$
\sigma\left(A+\mathbf{b} \mathbf{f}^{\top}\right)=\left\{\beta_{1}, \beta_{2}, \cdots, \beta_{n}\right\} \Longleftrightarrow \sigma(A(\mathbf{f}))=\left\{\beta_{1}, \beta_{2}, \cdots, \beta_{n}\right\} .
$$

In the above, note that for each given $\mathbf{b}$ the matrix $A_{\mathbf{b}}$ is known, and hence values of $\gamma_{i}$ are also known. To solve the ISVPrk for the case of $F=\mathbf{b f}^{\top}$, it suffices 
to determine the values of $f_{1}, \cdots, f_{n}$. Toward that end, we have the following necessary and sufficient condition.

Lemma 2.1. Given any fixed $\mathbf{b} \in \mathbb{R}^{m}$, there exists a vector $\mathbf{f} \in \mathbb{R}^{n}$ such that

$$
\sigma(A(\mathbf{f}))=\left\{\beta_{1}, \beta_{2}, \cdots, \beta_{n}\right\}
$$

if and only if

$$
\beta_{i} \geq \gamma_{i} \geq \beta_{i+1}, \quad i=1,2, \cdots, n
$$

where $\beta_{n+1}:=0$.

Proof. The necessity of the interlacing inequality (2.4) is a well-known property of singular value decompositions. See, for example, [12.

To prove the sufficiency, assume that the interlacing inequality (2.4) holds. Observe first that

$$
\mathcal{A}_{\mathbf{f}}:=A(\mathbf{f}) A(\mathbf{f})^{\top}=\left[\begin{array}{cccccc}
\sum_{i=1}^{n} f_{i}^{2} & f_{1} \gamma_{1} & f_{2} \gamma_{2} & \cdots & f_{n-1} \gamma_{n-1} & f_{n} \gamma_{n} \\
f_{1} \gamma_{1} & \gamma_{1}^{2} & & & & \\
f_{2} \gamma_{2} & & \gamma_{2}^{2} & & & \\
\vdots & & & \ddots & & \\
f_{n-1} \gamma_{n-1} & & & & \gamma_{n-1}^{2} & \\
f_{n} \gamma_{n} & & & & & \gamma_{n}^{2}
\end{array}\right]
$$

is a bordered matrix in $\mathbb{R}^{(n+1) \times(n+1)}$. As such, we now describe a way to construct a vector $\mathbf{f} \in \mathbb{R}^{n}$ such that (2.3) holds true. Our approach is a modification of a technique developed earlier for the Jacobi inverse eigenvalue problems [2]. Because

$$
\sigma(A(\mathbf{f}))=\left\{\beta_{1}, \cdots, \beta_{n}\right\} \Longleftrightarrow \lambda\left(\mathcal{A}_{\mathbf{f}}\right)=\left\{\beta_{1}^{2}, \cdots, \beta_{n}^{2}, 0\right\},
$$

finding a vector $\mathbf{f} \in \mathbb{R}^{n}$ such that (2.3) holds is equivalent to proving the identity

$$
p(\mu):=\mu \prod_{i=1}^{n}\left(\mu-\beta_{i}^{2}\right)-\operatorname{det}\left(\mu I-\mathcal{A}_{\mathbf{f}}\right) \equiv 0 .
$$

On one hand, note that

$$
p(\mu)=\left(\operatorname{trace}\left(\mathcal{A}_{\mathbf{f}}\right)-\sum_{i=1}^{n} \beta_{i}^{2}\right) \mu^{n}+\text { low degree terms in } \mu .
$$

So $p(\mu)$ is a polynomial of degree at most $n$ in $\mu$. On the other hand, we can expand the determinant of $\mathcal{A}_{\mathbf{f}}$ and thus expect the vector $\mathbf{f}$ to solve the equation

$$
\mu \prod_{j=1}^{n}\left(\mu-\beta_{j}^{2}\right)=\left(\mu-\sum_{i=1}^{n} f_{i}^{2}\right) \prod_{j=1}^{n}\left(\mu-\gamma_{j}^{2}\right)-\sum_{i=1}^{n}\left(\left(f_{i} \gamma_{i}\right)^{2} \prod_{\substack{j=1 \\ j \neq i}}^{n}\left(\mu-\gamma_{j}^{2}\right)\right)
$$

We shall divide our proof into four mutually exclusive cases.

Case 1. Assume that all $\gamma_{k}, k=1, \cdots n$, are distinct and nonzero. For each $k$, by setting $\mu=\gamma_{k}^{2}$ in (2.7), we obtain

$$
\gamma_{k}^{2} \prod_{j=1}^{n}\left(\gamma_{k}^{2}-\beta_{j}^{2}\right)=-\left(f_{k} \gamma_{k}\right)^{2} \prod_{\substack{j=1 \\ j \neq k}}^{n}\left(\gamma_{k}^{2}-\gamma_{j}^{2}\right) .
$$


Thus, $f_{k}^{2}$ is uniquely determined 11 by

$$
f_{k}^{2}=-\frac{\prod_{j=1}^{n}\left(\gamma_{k}^{2}-\beta_{j}^{2}\right)}{\prod_{\substack{j=1 \\ j \neq k}}^{n}\left(\gamma_{k}^{2}-\gamma_{j}^{2}\right)}, \quad k=1, \cdots, n .
$$

The interlacing property (2.4) guarantees that the right-hand side of (2.10) is nonnegative and hence real-valued $f_{k}$ can be defined. With this choice of $f_{1}, \cdots, f_{n}$, we see that $p(\mu)$ has $n+1$ zeros at $\mu=0, \gamma_{1}^{2}, \cdots, \gamma_{n}^{2}$, and hence $p(\mu) \equiv 0$.

Case 2. Assume that $\gamma_{1}>\cdots>\gamma_{t}>\gamma_{t+1}=\cdots=\gamma_{n}=0$ for some integer $t$. In this case, the interlacing inequality (2.4) implies that

$$
\beta_{t+2}=\cdots=\beta_{n}=0,
$$

and that the equality (2.7) is reduced to

$$
\begin{aligned}
\mu^{n-t} & \prod_{j=1}^{t+1}\left(\mu-\beta_{j}^{2}\right) \\
& =\mu^{n-t}\left\{\left(\mu-\sum_{i=1}^{n} f_{i}^{2}\right) \prod_{j=1}^{t}\left(\mu-\gamma_{j}^{2}\right)-\sum_{i=1}^{t}\left(\left(f_{i} \gamma_{i}\right)^{2} \prod_{\substack{j=1 \\
j \neq i}}^{t}\left(\mu-\gamma_{j}^{2}\right)\right)\right\} .
\end{aligned}
$$

It follows that

$$
\prod_{j=1}^{t+1}\left(\mu-\beta_{j}^{2}\right)=\left(\mu-\sum_{i=1}^{n} f_{i}^{2}\right) \prod_{j=1}^{t}\left(\mu-\gamma_{j}^{2}\right)-\sum_{i=1}^{t}\left(\left(f_{i} \gamma_{i}\right)^{2} \prod_{\substack{j=1 \\ j \neq i}}^{t}\left(\mu-\gamma_{j}^{2}\right)\right)
$$

By setting $\mu=\gamma_{k}^{2}$, we can determine that

$$
f_{k}^{2}=-\frac{\prod_{j=1}^{t+1}\left(\gamma_{k}^{2}-\beta_{j}^{2}\right)}{\gamma_{k}^{2} \prod_{\substack{j=1 \\ j \neq k}}^{t}\left(\gamma_{k}^{2}-\gamma_{j}^{2}\right)}, \quad k=1, \cdots, t .
$$

To obtain values for the remaining $f_{t+1}, \cdots, f_{n}$, observe that $\lambda\left(\mathcal{A}_{\mathbf{f}}\right)=\left\{\beta_{1}^{2}, \cdots, \beta_{t}^{2}\right.$, $\left.\beta_{t+1}^{2}, 0, \cdots, 0\right\}$. Computing the trace of $\mathcal{A}_{\mathbf{f}}$ yields the constraint that

$$
\sum_{i=1}^{t+1} \beta_{i}^{2}=\sum_{i=1}^{n} f_{i}^{2}+\sum_{i=1}^{n} \gamma_{i}^{2}=\sum_{i=1}^{n} f_{i}^{2}+\sum_{i=1}^{t} \gamma_{i}^{2} .
$$

\footnotetext{
${ }^{1}$ Incidentally, we have established an interesting equality which would be hard to prove in other context. By the fact that the sum of eigenvalues equals the trace, we have

$$
\sum_{i=1}^{n} f_{i}^{2}+\sum_{i=1}^{n} \gamma_{i}^{2}=\sum_{i=1}^{n} \beta_{i}^{2}
$$
}

The equality

$$
\sum_{i=1}^{n} \gamma_{i}^{2}-\sum_{i=1}^{n} \beta_{i}^{2}=\sum_{i=1}^{n} \frac{\prod_{j=1}^{n}\left(\gamma_{i}^{2}-\beta_{j}^{2}\right)}{\prod_{\substack{j=1 \\ j \neq i}}^{n}\left(\gamma_{i}^{2}-\gamma_{j}^{2}\right)}
$$

thus holds for any two sequences of numbers $\left\{\beta_{1}, \cdots, \beta_{n}\right\}$ and $\left\{\gamma_{1}, \cdots, \gamma_{n}\right\}$, if all $\gamma_{i}$ 's are distinct and nonzero, and (2.4) is satisfied. 
Therefore, the only condition imposed upon $f_{t+1}, \cdots, f_{n}$ is that

$$
\begin{aligned}
\sum_{i=t+1}^{n} f_{i}^{2} & =\beta_{t+1}^{2}+\left(\sum_{i=1}^{t} \beta_{i}^{2}-\sum_{i=1}^{t} \gamma_{i}^{2}\right)-\sum_{i=1}^{t} f_{i}^{2}, \\
& =\beta_{t+1}^{2}-\sum_{i=1}^{t} \frac{\prod_{\substack { j=1 \\
\begin{subarray}{c}{j=1 \\
j \neq i{ j = 1 \\
\begin{subarray} { c } { j = 1 \\
j \neq i } }\end{subarray}}^{t}\left(\gamma_{i}^{2}-\beta_{j}^{2}\right)}{\left.\prod_{i}^{2}-\gamma_{j}^{2}\right)}+\sum_{i=1}^{t} \frac{\prod_{j=1}^{t+1}\left(\gamma_{i}^{2}-\beta_{j}^{2}\right)}{\gamma_{i}^{2} \prod_{\substack{j=1 \\
j \neq i}}^{t}\left(\gamma_{i}^{2}-\gamma_{j}^{2}\right)} \\
& =\beta_{t+1}^{2}\left(1-\sum_{i=1}^{t} \frac{\prod_{j=1}^{t}\left(\gamma_{i}^{2}-\beta_{j}^{2}\right)}{\gamma_{i}^{2} \prod_{\substack{j=1 \\
j \neq i}}^{t}\left(\gamma_{i}^{2}-\gamma_{j}^{2}\right)}\right) .
\end{aligned}
$$

In the above we have employed the fact derived in (2.9) to arrive at the second equality. So long as $\mathbf{f} \in \mathbb{R}^{n}$ satisfy (2.14), the polynomial $p(\mu)$ in (2.6) is of degree at most $n-1$ in $\mu$ which, by (2.13), has $t$ nonzero roots at $\mu=\gamma_{k}^{2}, k=1, \ldots, t$ and one zero root with multiplicity $n-t$. It follows that $p(\mu) \equiv 0$.

Case 3. Assume that the set $\left\{\gamma_{1}, \gamma_{2}, \cdots, \gamma_{n}\right\}$ consists of $t$ distinct nonzero elements. For each $j=1, \cdots, t$, let $s_{j}$ denote the first index in the group such that

$$
\gamma_{s_{j}}=\gamma_{s_{j}+1}=\cdots=\gamma_{s_{j+1}-1},
$$

where $s_{t+1}=n+1$. The interlacing condition (2.4) enforces that

$$
\beta_{s_{j}+1}=\cdots=\beta_{s_{j+1}-1}=\gamma_{s_{j}} .
$$

Furthermore, the equality (2.7) becomes

$$
\begin{aligned}
& \mu\left\{\prod_{j=1}^{t}\left(\mu-\beta_{s_{j}}^{2}\right)\right\}\left\{\prod_{j=1}^{t}\left(\mu-\gamma_{s_{j}}^{2}\right)^{s_{j+1}-s_{j}-1}\right\}=\left\{\prod_{j=1}^{t}\left(\mu-\gamma_{s_{j}}^{2}\right)^{s_{j+1}-s_{j}-1}\right\} \\
& \times\left\{\left(\mu-\sum_{i=1}^{n} f_{i}^{2}\right) \prod_{j=1}^{t}\left(\mu-\gamma_{s_{j}}^{2}\right)-\sum_{i=1}^{t}\left(\left(f_{s_{i}}^{2}+\cdots+f_{s_{i+1}-1}^{2}\right) \gamma_{s_{i}}^{2} \prod_{\substack{j=1 \\
j \neq i}}^{t}\left(\mu-\gamma_{s_{j}}^{2}\right)\right)\right\} .
\end{aligned}
$$

After cancellation, we obtain that

$$
\begin{aligned}
\mu\left\{\prod_{j=1}^{t}\left(\mu-\beta_{s_{j}}^{2}\right)\right\} & =\left(\mu-\sum_{j=1}^{t} \sum_{i=s_{j}}^{s_{j+1}-1} f_{i}^{2}\right) \prod_{j=1}^{t}\left(\mu-\gamma_{s_{j}}^{2}\right) \\
& \left.-\sum_{i=1}^{t}\left(\sum_{s=s_{i}}^{s_{i}+1-1} f_{s}^{2}\right) \gamma_{s_{i}}^{2} \prod_{\substack{j=1 \\
j \neq i}}^{t}\left(\mu-\gamma_{s_{j}}^{2}\right)\right)
\end{aligned}
$$

Consequently, by setting $\mu=\gamma_{s_{k}}^{2}$, we obtain the following set of constraints:

$$
\sum_{s=s_{k}}^{s_{k+1}-1} f_{s}^{2}=-\frac{\prod_{\substack{j=1 \\ j \neq k}}^{t}\left(\gamma_{s_{k}}^{2}-\beta_{s_{j}}^{2}\right)}{\prod_{\substack{j=1 \\ j \neq k}}^{t}\left(\gamma_{s_{k}}^{2}-\gamma_{s_{j}}^{2}\right)}, \quad k=1, \cdots, t .
$$


Note that formula (2.17) is similar to formula (2.10) in Case 1. Using a similar argument, it can now be shown that $p(\mu) \equiv 0$ whenever $\mathbf{f}$ satisfies the condition (2.17).

Case 4. Assume that the set $\left\{\gamma_{1}, \gamma_{2}, \cdots, \gamma_{n}\right\}$ consists of $t+1$ distinct elements including one zero, that is, assume that

$$
\gamma_{s_{1}}>\gamma_{s_{2}}>\cdots>\gamma_{s_{t}}>\gamma_{s_{t+1}}=0
$$

where the indices $s_{j}$ are defined in the same way as those in Case 3. The interlacing inequality (2.4) implies that

$$
\begin{aligned}
& \beta_{s_{j}+1}=\cdots=\beta_{s_{j+1}-1}=\gamma_{s_{j}}, \\
& \beta_{s_{t+1}+1}=\cdots=\beta_{n}=0,
\end{aligned}
$$

and the equality (2.7) becomes

$$
\begin{aligned}
& \mu^{n-s_{t+1}+1}\left\{\prod_{j=1}^{t+1}\left(\mu-\beta_{s_{j}}^{2}\right)\right\}\left\{\prod_{j=1}^{t}\left(\mu-\gamma_{s_{j}}^{2}\right)^{s_{j+1}-s_{j}-1}\right\} \\
& =\mu^{n-s_{t+1}+1}\left\{\prod_{j=1}^{t}\left(\mu-\gamma_{s_{j}}^{2}\right)^{s_{j+1}-s_{j}-1}\right\} \\
& \left.\times\left\{\left(\mu-\sum_{i=1}^{n} f_{i}^{2}\right) \prod_{j=1}^{t}\left(\mu-\gamma_{s_{k}}^{2}\right)-\sum_{i=1}^{t}\left(f_{s_{i}}^{2}+\cdots+f_{s_{i+1}-1}^{2}\right) \gamma_{s_{i}}^{2} \prod_{\substack{j=1 \\
j \neq i}}^{t}\left(\mu-\gamma_{s_{j}}^{2}\right)\right)\right\},
\end{aligned}
$$

which is further reduced to

$$
\begin{aligned}
& \prod_{j=1}^{t+1}\left(\mu-\beta_{s_{j}}^{2}\right) \\
& \quad=\left(\mu-\sum_{i=1}^{n} f_{i}^{2}\right) \prod_{j=1}^{t}\left(\mu-\gamma_{s_{j}}^{2}\right)-\sum_{i=1}^{t}\left(\left(f_{i_{k}}^{2}+\cdots+f_{i_{k+1}-1}^{2}\right) \gamma_{s_{i}}^{2} \prod_{\substack{j=1 \\
j \neq i}}^{t}\left(\mu-\gamma_{s_{j}}^{2}\right)\right) .
\end{aligned}
$$

So, we have by setting $\mu=\gamma_{s_{k}}^{2}$ that

$$
\sum_{s=s_{k}}^{s_{k+1}-1} f_{s}^{2}=-\frac{\prod_{j=1}^{t+1}\left(\gamma_{s_{k}}^{2}-\beta_{s_{j}}^{2}\right)}{\gamma_{s_{k}}^{2} \prod_{\substack{j=1 \\ j \neq k}}^{t}\left(\gamma_{s_{k}}^{2}-\gamma_{s_{j}}^{2}\right)}, \quad k=1, \cdots, t
$$


Finally, the constraint imposed upon $\sum_{s=s_{t+1}}^{n} f_{s}^{2}$ can be obtained by the trace condition (2.8). That is, we obtain by using identity (2.9) that

$$
\begin{aligned}
\sum_{s=s_{t+1}}^{n} f_{s}^{2} & =\sum_{i=1}^{n} \beta_{i}^{2}-\sum_{i=1}^{n} \gamma_{i}^{2}-\sum_{i=1}^{t} \sum_{s=s_{i}}^{s_{i+1}-1} f_{s}^{2} \\
& =\beta_{s_{t+1}}^{2}+\left(\sum_{i=1}^{t} \beta_{s_{i}}^{2}-\sum_{i=1}^{t} \gamma_{s_{i}}^{2}\right)-\sum_{i=1}^{t} \sum_{s=s_{i}}^{s_{i+1}-1} f_{s}^{2} \\
& =\beta_{s_{t+1}}^{2}-\sum_{i=1}^{t} \frac{\prod_{j=1}^{t}\left(\gamma_{s_{i}}^{2}-\beta_{s_{j}}^{2}\right)}{\prod_{\substack{j=1 \\
j \neq i}}^{t}\left(\gamma_{s_{i}}^{2}-\gamma_{s_{j}}^{2}\right)}+\sum_{i=1}^{t} \frac{\prod_{j=1}^{t+1}\left(\gamma_{s_{i}}^{2}-\beta_{s_{j}}^{2}\right)}{\gamma_{s_{i}}^{2} \prod_{\substack{j=1 \\
j \neq i}}^{t}\left(\gamma_{s_{i}}^{2}-\gamma_{s_{j}}^{2}\right)} \\
& =\beta_{s_{t+1}}^{2}\left(1-\sum_{i=1}^{t} \frac{\prod_{j=1}^{t}\left(\gamma_{s_{i}}^{2}-\beta_{s_{j}}^{2}\right)}{\gamma_{s_{i}}^{2}\left[\prod_{\substack{j=1 \\
j \neq i}}^{t}\left(\gamma_{s_{i}}^{2}-\gamma_{s_{j}}^{2}\right)\right]}\right) .
\end{aligned}
$$

Now using similar arguments as in Cases 1, 2, and 3, we can prove (2.3) so long as f satisfies both (2.19) and (2.20).

With all four cases considered, the sufficiency of (2.4) is established. The proof is now completed.

Recall that the values of $\gamma_{i}$ referred to in Lemma 2.1 are determined by the orthogonal transformation $Q_{\mathbf{b}}$ in (2.1) which, in turn, depends on the vector $\mathbf{b}$. This relationship indicates that if the vector $\mathbf{b}$ is changed, then the interlacing inequality (2.4) will also be changed. Recall also that if

$$
\sigma(A)=\left\{\alpha_{1}, \cdots, \alpha_{n}\right\}, \quad \alpha_{1} \geq \alpha_{2} \geq \cdots \geq \alpha_{n},
$$

then the $\gamma_{i}$ 's corresponding to any $\mathbf{b}$ must satisfy the inequalities

$$
\alpha_{i} \geq \gamma_{i} \geq \alpha_{i+1}, \quad i=1, \cdots, n,
$$

with $\alpha_{n+1}=0$. We are thus motivated to consider using $\mathbf{b}$ as a parameter to control the singular values. The following result is particularly interesting.

Lemma 2.2. Let $A \in \mathbb{R}^{m \times n}(m \geq n)$ be given and fixed. Corresponding to any values $\gamma_{i}, i=1, \cdots, n$, satisfying the interlacing inequality (2.21) where $\gamma_{n}=0$ if $m=n$, there exist a unit vector $\mathbf{b} \in \mathbb{R}^{m}$ and an orthogonal matrix $Q_{\mathbf{b}} \in \mathbb{R}^{m \times m}$ such that

$$
Q_{\mathbf{b}}^{\top} \mathbf{b}=\left[\begin{array}{l}
1 \\
\mathbf{0}
\end{array}\right]
$$

and

$$
Q_{\mathbf{b}}^{\top} A=\left[\begin{array}{c}
\mathbf{a}_{\mathbf{b}}^{\top} \\
A_{\mathbf{b}}
\end{array}\right]
$$

with

$$
\sigma\left(A_{\mathbf{b}}\right)= \begin{cases}\left\{\gamma_{1}, \cdots, \gamma_{n-1}\right\}, & \text { if } m=n \\ \left\{\gamma_{1}, \cdots, \gamma_{n}\right\}, & \text { if } m>n\end{cases}
$$


Proof. Depending on whether $m=n$ or $m>n$, define $\tilde{A} \in \mathbb{R}^{m \times n}$ by

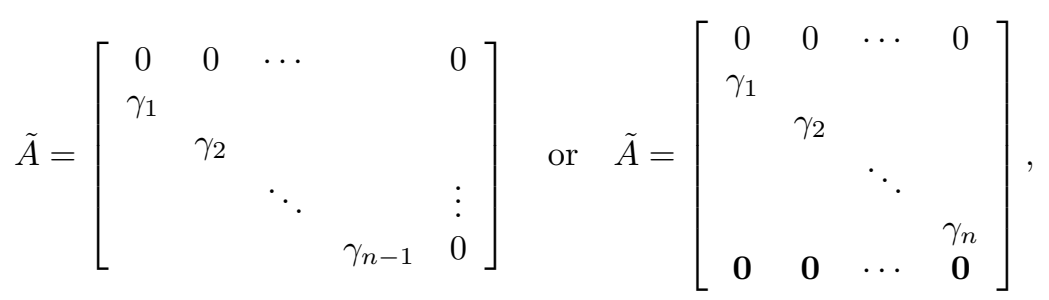

where the last row of $\mathbf{0}$ 's are used, if $m>n+1$, to pad the row dimension $m$. Because of the interlacing inequality (2.21), by Lemma 2.1 with $\tilde{\mathbf{b}}=\left[\begin{array}{l}1 \\ \mathbf{0}\end{array}\right] \in \mathbb{R}^{m}$, there exists a column vector $\mathbf{c} \in \mathbb{R}^{n}$ such that

$$
\sigma\left(\tilde{A}+\tilde{\mathbf{b}} \mathbf{c}^{\top}\right)=\left\{\alpha_{1}, \alpha_{2}, \cdots, \alpha_{n}\right\} .
$$

Let the singular value decompositions of $A$ and $\tilde{A}+\tilde{\mathbf{b}} \mathbf{c}^{\top}$ be denoted as $A=U_{1} \Sigma V_{1}^{\top}$ and $\tilde{A}+\tilde{\mathbf{b}} \mathbf{c}^{\top}=U_{2} \Sigma V_{2}^{\top}$, respectively. Define

$$
Q_{\mathbf{b}}:=U_{1} U_{2}^{\top} \quad \text { and } \quad \mathbf{b}:=Q_{\mathbf{b}} \tilde{\mathbf{b}} .
$$

The partition

$$
Q_{\mathbf{b}}^{\top} A=\left(\tilde{A}+\tilde{\mathbf{b}} \mathbf{c}^{\top}\right)\left(V_{2} V_{1}^{\top}\right)=\left[\begin{array}{c}
\mathbf{a}_{\mathbf{b}}^{\top} \\
A_{\mathbf{b}}
\end{array}\right]
$$

has the properties that

$$
\mathbf{a}_{\mathbf{b}}=A^{\top} \mathbf{b} \quad\left(=V_{1} V_{2}^{\top} \mathbf{c}\right)
$$

and

$$
A_{\mathbf{b}}=\left[\begin{array}{ccccc}
\gamma_{1} & & & & 0 \\
& \gamma_{2} & & & \\
& & \ddots & & \vdots \\
& & & \gamma_{n-1} & 0
\end{array}\right] V_{2} V_{1}^{\top} \quad \text { or }\left[\begin{array}{cccc}
\gamma_{1} & & & \\
& \gamma_{2} & & \\
& & \ddots & \\
& & & \gamma_{n} \\
0 & 0 & \cdots & 0
\end{array}\right] V_{2} V_{1}^{\top} .
$$

The assertion therefore is proved.

We are now able to completely characterize the solvability of ISVPrk with the following result.

Theorem 2.3. The following three statements are equivalent:

1. The ISVPrk with $\ell=1$ is solvable.

2. For each $i=1, \cdots, n$, there exists a value $\gamma_{i}$ satisfying both inequalities

$$
\begin{gathered}
\alpha_{i} \geq \gamma_{i} \geq \alpha_{i+1}, \\
\beta_{i} \geq \gamma_{i} \geq \beta_{i+1},
\end{gathered}
$$

where $\alpha_{n+1}:=0$ and $\beta_{n+1}:=0$.

3. For each $i=1, \cdots, n-1$,

$$
\beta_{i+1} \leq \alpha_{i} \quad \text { and } \quad \alpha_{i+1} \leq \beta_{i} .
$$

Proof. By keeping the ordering $\alpha_{1} \geq \alpha_{2} \geq \cdots \geq \alpha_{n}$ and $\beta_{1} \geq \beta_{2} \geq \cdots \geq \beta_{n}$, the equivalence of statements 2 and 3 is obvious. We only need to show the equivalence of statements 1 and 2 . 
Assume that the ISVPrk has a rank-one solution $F \in \mathbb{R}^{m \times n}$. There exists an orthogonal matrix $Q_{F}$ such that

$$
Q_{F}^{\top} F=\left[\begin{array}{l}
\mathbf{f}^{\top} \\
\mathbf{O}
\end{array}\right]
$$

where $\mathbf{f} \in \mathbb{R}^{n}$ and $\mathbf{O}$ is a zero matrix in $\mathbb{R}^{(m-1) \times n}$. Write

$$
Q_{F}^{\top} A=\left[\begin{array}{l}
\mathbf{a}_{\mathbf{f}}^{\top} \\
A_{F}
\end{array}\right]
$$

where $\mathbf{a}_{\mathbf{f}} \in \mathbb{R}^{n}$ and $A_{F} \in \mathbb{R}^{(m-1) \times n}$. Let

$$
\sigma\left(A_{F}\right)= \begin{cases}\left\{\gamma_{1}, \gamma_{2}, \cdots, \gamma_{n-1}\right\} & \text { if } m=n, \\ \left\{\gamma_{1}, \gamma_{2}, \cdots, \gamma_{n}\right\}, & \text { if } m>n,\end{cases}
$$

with the descending order $\gamma_{1} \geq \gamma_{2} \geq \cdots$. Then by the fact that $A_{F}$ is a submatrix of both $Q_{F}^{\top} A$ and $Q_{F}^{\top}(A+F)$, the singular values of $A_{F}$ interlace those of both $Q_{F}^{\top} A$ and $Q_{F}^{\top}(A+F)$, giving rise to the inequalities (2.23) and (2.24), respectively. We thus have proved that statement 1 implies statement 2 .

To show the converse, assume first that the interlacing inequality (2.23) holds. By Lemma 2.2, there exist a unit vector $\mathbf{b} \in \mathbb{R}^{m}$ and orthogonal matrix $Q_{\mathbf{b}} \in \mathbb{R}^{m \times m}$ such that $Q_{\mathbf{b}}^{\top} \mathbf{b}=\left[\begin{array}{l}1 \\ \mathbf{0}\end{array}\right], Q_{\mathbf{b}}^{\top} A=\left[\begin{array}{l}\mathbf{a}_{\mathbf{b}}^{\top} \\ A_{\mathbf{b}}\end{array}\right]$, and $\sigma\left(A_{\mathbf{b}}\right)$ is precisely as described in (2.22). By the interlacing inequality (2.24) and Lemma 2.1, we then obtain a vector $\mathbf{f} \in \mathbb{R}^{n}$ such that

$$
\sigma\left(A+\mathbf{b f}^{\top}\right)=\left\{\beta_{1}, \beta_{2}, \cdots, \beta_{n}\right\}
$$

Therefore, the ISVPrk is solved by defining $F=\mathbf{b f}^{\top}$.

It is worthy to point out that the $\alpha_{i}$ 's and $\beta_{i}$ 's in Theorem 2.3 do not necessarily satisfy any interlacing property. The feasible range for the case $n=3$ is illustrated in Figure 1. Note that $\beta_{2}$ can be any value between $\alpha_{1}$ and $\alpha_{3}$ so long as $\beta_{1} \geq$ $\beta_{2} \geq \beta_{3}$.

We conclude this section with an interesting observation characterizing the class of special matrices $A$ for which the singular values cannot be reassigned by any rank-one matrices.

The following result is a corollary of Theorem 2.3

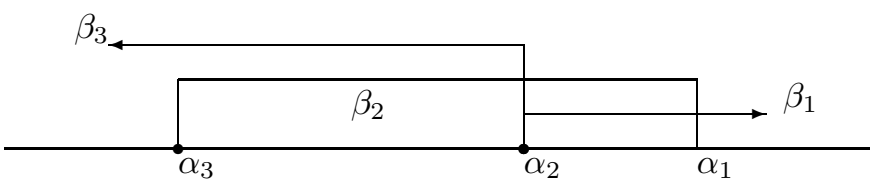

Figure 1 . Feasible range of $\alpha_{i}$ 's and $\beta_{i}$ 's for the case $n=3$. 
Corollary 2.4. Let the multiplicity of all distinct singular values $\alpha_{1}(A), \cdots$, $\alpha_{t}(A)$ of $A$ be denoted by $s_{1}, \cdots, s_{t}$. Then

$$
\bigcap_{\operatorname{rank}(F) \leq 1} \sigma(A+F)=\left\{\alpha_{k}(A) \text { with algebraic multiplicity }\left(s_{k}-2\right) \mid s_{k}>2,1 \leq k \leq t\right\} .
$$

Hence,

$$
\bigcap_{\operatorname{rank}(F) \leq 1} \sigma(A+F)=\emptyset \quad \Longleftrightarrow \quad s_{k} \leq 2, k=1, \cdots, t
$$

\section{MAin RESUlt}

We are now ready to deal with the general ISVPrk. The following result includes Theorem 2.3 as a special case, in that the "gap" between the original singular values $\alpha_{i}$ and the desirable singular values $\beta_{i}$ is separated by the rank number $\ell$.

Theorem 3.1. The ISVPrk is solvable if and only if for each $i=1, \cdots, n-\ell$,

$$
\beta_{i+\ell} \leq \alpha_{i} \quad \text { and } \quad \alpha_{i+\ell} \leq \beta_{i}
$$

Proof. We shall prove our result by mathematical induction. Note that we have already established the case for $\ell=1$ in Theorem 2.3. Assume that the assertion in Theorem 3.1 is true for $\ell=k$. We want to establish the case $\ell=k+1 \leq n$.

We first argue the necessity. Any $F \in \mathbb{R}^{m \times n}$ with $\operatorname{rank}(F) \leq k+1$ can be factorized as

$$
F=F_{1}+F_{2},
$$

with $\operatorname{rank}\left(F_{1}\right) \leq k$ and $\operatorname{rank}\left(F_{2}\right) \leq 1$. Denote

$$
\sigma\left(A+F_{1}\right)=\left\{\gamma_{1}, \gamma_{2}, \cdots, \gamma_{n}\right\}
$$

Then, by assumption, it must be that for each $i=1, \cdots, n-k$

$$
\gamma_{i+k} \leq \alpha_{i} \quad \text { and } \quad \alpha_{i+k} \leq \gamma_{i} .
$$

In addition, using Theorem 2.3, we have that for each $i=1, \cdots, n-1$

$$
\beta_{i+1} \leq \gamma_{i} \text { and } \gamma_{i+1} \leq \beta_{i}
$$

Together, we see for each $i=1, \cdots, n-k-1$ it must be true that

$$
\beta_{i+k+1} \leq \alpha_{i} \quad \text { and } \quad \alpha_{i+k+1} \leq \beta_{i} .
$$

We then argue the sufficiency. Note that

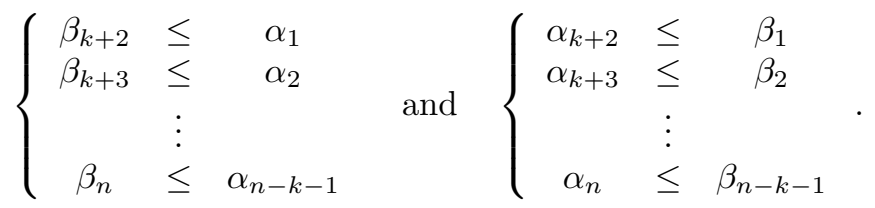

It is a matter of inspection that there exist $\gamma_{i}, i=1, \cdots, n$, with

$$
\gamma_{1} \geq \gamma_{2} \geq \cdots \geq \gamma_{n}
$$


such that

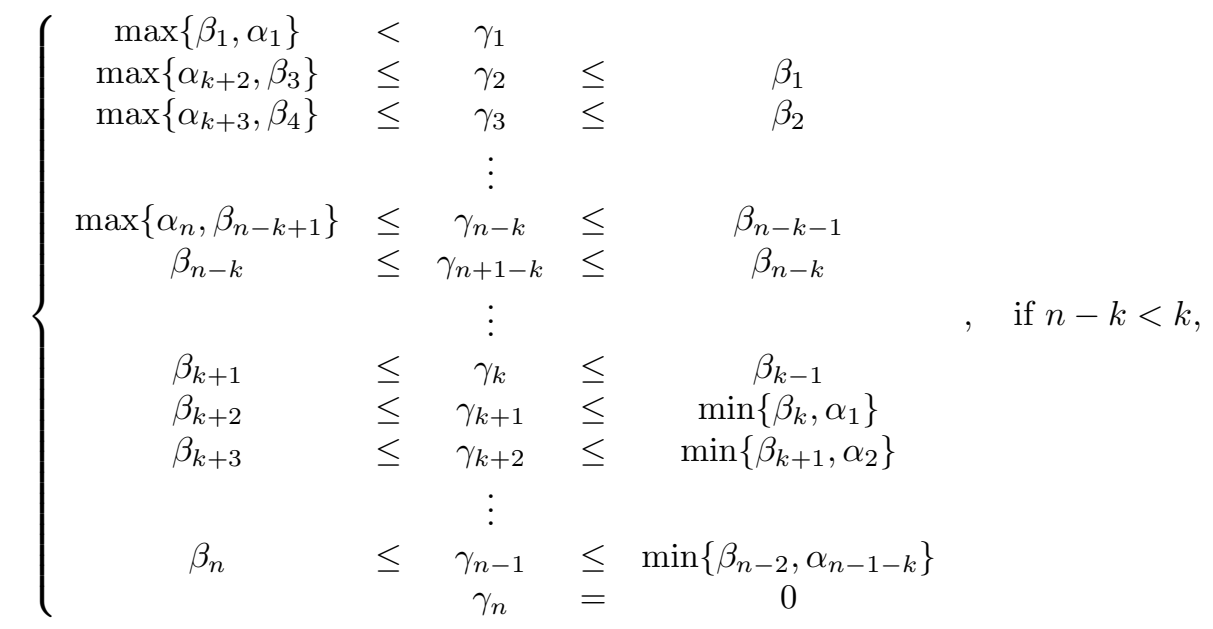

or

$$
\begin{aligned}
& \left\{\begin{array}{c}
\max \left\{\beta_{1}, \alpha_{1}\right\}<\gamma_{1} \\
\max \left\{\alpha_{k+2}, \beta_{3}\right\} \leq \gamma_{2} \leq \\
\max \left\{\alpha_{k+3}, \beta_{4}\right\} \leq \gamma_{3} \leq \beta_{1}
\end{array}\right. \\
& \begin{array}{c}
\max \left\{\alpha_{n}, \beta_{n-k+1}\right\} \\
\beta_{k+2} \\
\beta_{k+3} \gamma_{n-k} \leq \gamma_{k+1} \leq \beta_{n-k-1} \\
\min \left\{\beta_{k}, \alpha_{1}\right\}
\end{array} \quad, \quad \text { if } n-k=k \\
& \beta_{k+3} \leq \gamma_{k+2} \leq \min \left\{\beta_{k+1}, \alpha_{2}\right\} \\
& \beta_{n} \leq \begin{array}{ccc}
\gamma_{n-1} & \leq & \min \left\{\beta_{n-2}, \alpha_{n-1-k}\right\} \\
\gamma_{n} & = & 0
\end{array}
\end{aligned}
$$

or

$$
\left\{\begin{array}{ccccc}
\max \left\{\alpha_{1}, \beta_{1}\right\} & < & \gamma_{1} & & \\
\max \left\{\alpha_{k+2}, \beta_{3}\right\} & \leq & \gamma_{2} & \leq & \beta_{1} \\
\max \left\{\alpha_{k+3}, \beta_{4}\right\} & \leq & \gamma_{3} & \leq & \beta_{2} \\
& & \vdots & & \\
\max \left\{\alpha_{2 k}, \beta_{k+1}\right\} & \leq & \gamma_{k} & \leq & \beta_{k-1} \\
\max \left\{\alpha_{2 k+1}, \beta_{k+2}\right\} & \leq & \gamma_{k+1} & \leq & \min \left\{\alpha_{1}, \beta_{k}\right\} \\
\max \left\{\alpha_{2 k+2}, \beta_{k+3}\right\} & \leq & \gamma_{k+2} & \leq & \min \left\{\alpha_{2}, \beta_{k+1}\right\} \\
& & \vdots & & \\
\max \left\{\alpha_{n}, \beta_{k+J+2}\right\} & \leq & \gamma_{k+J+1} & \leq & \min \left\{\alpha_{J+1}, \beta_{k+J}\right\} \\
\beta_{k+J+3} & \leq & \gamma_{k+J+2} & \leq & \min \left\{\alpha_{J+2}, \beta_{k+J+1}\right\} \\
\beta_{k+J+4} & \leq & \gamma_{k+J+3} & \leq & \min \left\{\alpha_{J+3}, \beta_{k+J+2}\right\} \\
& & & & \\
\beta_{n} & \leq & \gamma_{n-1} & \leq & \min \left\{\alpha_{n-k-1}, \beta_{n-2}\right\} \\
& & & \gamma_{n} & =
\end{array}\right.
$$

with $J=(n-k)-(k+1)$. These values of $\gamma_{i}, i=1, \cdots, n$, satisfy

$$
\gamma_{i+k} \leq \alpha_{i}, \quad \alpha_{i+k} \leq \gamma_{i}, \quad i=1, \cdots, n-k,
$$


and

$$
\beta_{i+1} \leq \gamma_{i}, \quad \gamma_{i+1} \leq \beta_{i}, \quad i=1, \cdots, n-1
$$

By the inductive assumption, there exists a matrix $F_{1} \in \mathbb{R}^{m \times n}$ such that

$$
\operatorname{rank}\left(F_{1}\right) \leq k, \quad \sigma\left(A+F_{1}\right)=\left\{\gamma_{1}, \gamma_{2}, \cdots, \gamma_{n}\right\} .
$$

Furthermore, by Theorem 2.3. there exists a matrix $F_{2} \in \mathbb{R}^{m \times n}$ such that

$$
\operatorname{rank}\left(F_{2}\right) \leq 1, \quad \sigma\left(\left(A+F_{1}\right)+F_{2}\right)=\left\{\beta_{1}, \beta_{2}, \cdots, \beta_{n}\right\} .
$$

Hence, if we define

$$
F=F_{1}+F_{2},
$$

then $F$ satisfies

$$
\operatorname{rank}(F) \leq \operatorname{rank}\left(F_{1}\right)+\operatorname{rank}\left(F_{2}\right) \leq k+1, \quad \sigma(A+F)=\left\{\beta_{1}, \beta_{2}, \cdots, \beta_{n}\right\} .
$$

This completes the proof of sufficiency.

By the mathematical induction principle, Theorem 3.1 is true for any $1 \leq \ell \leq$ $n$.

A consequence of Theorem 3.1 is worth mentioning. If condition (3.1) holds, then there exists a matrix $F$ with $\operatorname{rank}(F) \leq \ell$ that solves the ISVPrk. But, if condition (3.1) holds and if for at least one $i$ such that $\beta_{i+\ell-1}>\alpha_{i}$ or $\alpha_{i+\ell-1}>\beta_{i}$, then no matrix $F$ with $\operatorname{rank}(F) \leq \ell-1$ can solve the ISVPrk. In other words, $\ell$ is the minimal rank for all $F$ that solves the ISVPrk.

The necessary condition of Theorem 3.1 is related to the classical Weyl inequality for singular values of sums of matrices. See, for example, the earlier paper [1] and the seminal books 16 and 23 . However, we believe that the fact that the necessary condition is also sufficient is new and of significant importance. The simplicity of condition (3.1) is also quite pleasant.

\section{NumericAl ALGORITHM}

The proofs given above can be implemented as numerical means to compute a solution for the ISVPrk. For clarity, we represent the procedures in algorithmic format.

Algorithm 4.1 (Singular Value Reassignment with Rank One Update). Given a matrix $A \in \mathbb{R}^{m \times n}$ with $m \geq n$ and a set of nonnegative numbers $\beta_{1} \geq \cdots \geq \beta_{n}$, the following steps check and find vectors $\mathbf{b} \in \mathbb{R}^{m}$ and $\mathbf{f} \in \mathbb{R}^{n}$ such that $\sigma\left(A+\mathbf{b f}^{\top}\right)=$ $\left\{\beta_{1}, \cdots, \beta_{n}\right\}$ :

1. Compute the singular value decomposition

$$
A=U_{1} \Sigma V_{1}^{\top}
$$

and denote $\sigma(A)=\left\{\alpha_{1}, \cdots, \alpha_{n}\right\}$ with $\alpha_{1} \geq \cdots \geq \alpha_{n}$.

2. For $i=1, \cdots, n-1$, check to see if

$$
\beta_{i+1} \leq \alpha_{i} \quad \text { and } \quad \alpha_{i+1} \leq \beta_{i} .
$$

If not, stop.

3. For $i=1, \cdots, n-1$, define

$$
\gamma_{i}:=\frac{\min \left\{\alpha_{i}, \beta_{i}\right\}+\max \left\{\alpha_{i+1}, \beta_{i+1}\right\}}{2}
$$


and

$$
\gamma_{n}:= \begin{cases}0, & \text { if } m=n, \\ \frac{\min \left\{\alpha_{n}, \beta_{n}\right\}}{2}, & \text { otherwise. }\end{cases}
$$

4. If $\gamma_{1}>\cdots>\gamma_{n}>0$, define for each $k=1, \cdots, n$

$$
c_{k}:=\sqrt{-\frac{\prod_{j=1}^{n}\left(\gamma_{k}^{2}-\alpha_{j}^{2}\right)}{\prod_{\substack{j=1 \\ j \neq k}}^{n}\left(\gamma_{k}^{2}-\gamma_{j}^{2}\right)}}
$$

else modify the $c_{k}$ 's according to the remaining three cases discussed in Lemma 2.1

5. Define

$$
\hat{A}:= \begin{cases}{\left[\mathbf{c}^{\top} ; \operatorname{diag}\left(\gamma_{1}, \cdots, \gamma_{n-1}\right), \operatorname{zeros}(m-1,1)\right],} & \text { if } m=n, \\ {\left[\mathbf{c}^{\top} ; \operatorname{diag}\left(\gamma_{1}, \cdots, \gamma_{n}\right)\right],} & \text { if } m=n+1, \\ {\left[\mathbf{c}^{\top} ; \operatorname{diag}\left(\gamma_{1}, \cdots, \gamma_{n}\right) ; \operatorname{zeros}(m-n-1, n)\right],} & \text { otherwise. }\end{cases}
$$

6. Compute the singular value decomposition

$$
\hat{A}:=U_{2} \Sigma V_{2}^{\top} \text {. }
$$

7. Define

$$
\begin{aligned}
\mathbf{b} & :=U_{1} U_{2}(1,:)^{\top}, \\
V_{\mathbf{b}} & :=V_{1} V_{2}^{\top}, \\
\mathbf{a}_{\mathbf{b}} & :=A^{\top} \mathbf{b}\left(\text { or } V_{\mathbf{b}} \mathbf{c}\right) .
\end{aligned}
$$

8. If $\gamma_{1}>\cdots>\gamma_{n}>0$, define for each $k=1, \cdots, n$

$$
\hat{f}_{k}:=\sqrt{-\frac{\prod_{j=1}^{n}\left(\gamma_{k}^{2}-\beta_{j}^{2}\right)}{\prod_{\substack{j=1 \\ j \neq k}}^{n}\left(\gamma_{k}^{2}-\gamma_{j}^{2}\right)}}
$$

else modify the $\hat{f}_{k}$ 's according to the remaining three cases discussed in Lemma 2.1,

\section{Define}

$$
\mathbf{f}:=V_{\mathbf{b}} \hat{\mathbf{f}}-\mathbf{a}_{\mathbf{b}} .
$$

We remark that the selection of midpoints in step 3 of Algorithm 4.1 is but one safe way to ensure conditions (2.23) and (2.24). Other choices are certainly possible. Also, we have to point out that details of a mathematically equivalent but numerically more stable way of computing the ratios in $\mathbf{c}$ and $\hat{\mathbf{f}}$ have been discussed elsewhere in the context of Jacobi inverse eigenvalue problems [9, 13] which we shall not elaborate here. If so desired, one simply needs to replace formulas in step 4 and step 8 by the more stable formulation.

Once a rank-one update algorithm is available, it is important to realize that the entire induction process described in Theorem 3.1 can easily be implemented in any programming language that supports a routine to call itself recursive. See discussions of similar implementations in, for example, [5, 6. The main feature in the routine should be a single divide-and-conquer mechanism that can be demonstrated by the pseudo-code listed in Table 1 .

As the routine svd_update is calling itself recursively when ell is not one, the rank of the problem is reduced by one successively and the current data are saved 
TABLE 1. A pseudo-MATLAB program for the recursive algorithm.

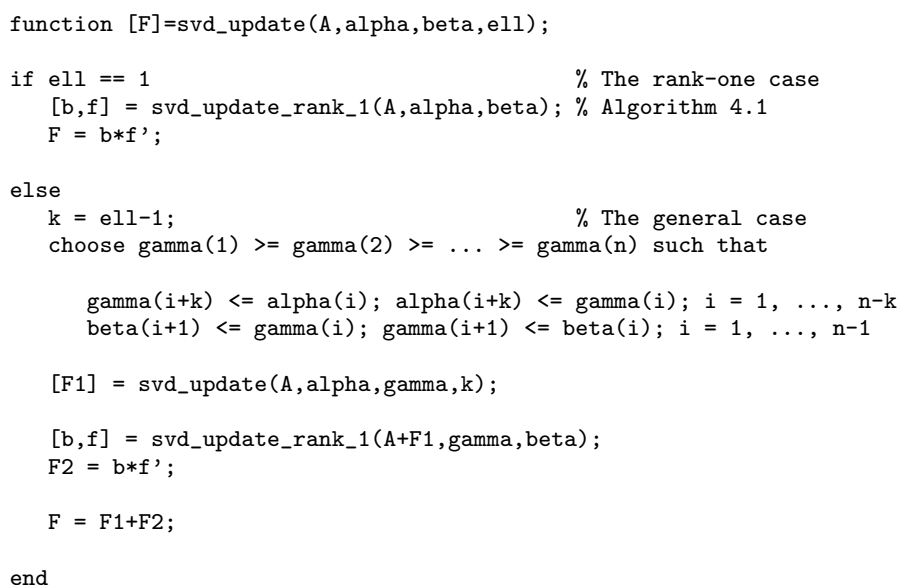

internally. This is the "dividing" process. Once ell has reached the bottom, that is, when $\mathrm{ell}=1$, the process is reversed and begins to build up matrices $\mathrm{F} 1$ followed by F2 layer by layer. This is the "conquering" process. The final output F is the desired solution to the ISVPrk.

\section{Conclusion}

We have provided a rigorous theoretic basis for the singular value reassignment problem. The simple necessary and sufficient condition (3.1) completely settles the issue of solvability for the ISVPrk. Our proof is constructive so it can be exploited to provide a possible means for computing the solution numerically. Using the rank-one case as the building block, the algorithm features a divide-and-conquer scheme. The numerical procedure as it stands now, particularly Algorithm 4.1, might not be stable when there are close-by singular values. Remedies are available in the literature, but this paper mainly concentrates on the general ideas.

\section{ACKNOWLEDGMENT}

The original idea of the ISVPrk with rank-one update was brought to our attention by Wojciech A. Tadej.

\section{REFERENCES}

[1] A. R. Amir-Moez, Extreme properties of eigenvalues of a hermitian transformation and singular values of the sum and product of linear transformations, Duke Math. J., 23(1955), 463-476. MR0079564 (18,105j)

[2] D. Boley and G. H. Golub, A survey of matrix inverse eigenvalue problem, Inverse Problems, 3(1987), 595-622. MR0928047 (89m:65036)

[3] C. I. Byrnes, Pole placement by output feedback, in Three Decades of Mathematical Systems Theory, Lecture Notes in Control and Information Sciences, Springer-Verlag, 135(1989), 3178. MR.1025786 (90k:93001)

[4] M. T. Chu, Numerical methods for inverse singular value problems, SIAM J. Numer. Anal., 29(1992), 885-903. MR.1163362 (93a:65048) 
[5] M. T. Chu, On constructing matrices with prescribed singular values and diagonal elements, Linear Alg. Appl., 288(1999), 11-22. MR1670590(2000a:15010)

[6] M. T. Chu, A fast recursive algorithm for constructing matrices with prescribed eigenvalues and singular values, SIAM J. Numer. Anal., 37(2000), 1004-1020. MR.1749246 (2001d:65044)

[7] M. T. Chu and G. H. Golub, Structured inverse eigenvalue problems, Acta Numerica, 12(2002), 1-71. MR2008966 (2005b:65040)

[8] M. T. Chu, R. E. Funderlic, and R. J. Plemmons, Structured low-rank approximation, Linear Alg. Appl., 366(2003), 157-172. MR1987719 (2004e:15001)

[9] C. de Boor and G. H. Golub, The numerically stable reconstruction of a Jacobi matrix from spectral data, Linear Alg. Appl., 21(1978), 245-260. MR0504044(80i:15007)

[10] G. N. de Oliveira, Matrices with prescribed principal elements and singular values, Canada Math. Bull., 14(1971), 247-249. MR0311686 (47:248)

[11] I. Gohberg, M. Kaashoek, and F. van Schagen, Partially specified matrices and operators: classification, completion, applications, Operator Theory: Advances and Applications Series, vol. 79, Birkhäuser Verlag, Basel, 1995. MR1409814 (97i:47002)

[12] G. H. Golub and C. F. Van Loan. Matrix Computations, The Johns Hopkins University Press, Baltimore, Maryland, 3rd edition, 1996. MR.1417720 (97g:65006)

[13] W. B. Gragg and W. J. Harrod, The numerically stable reconstruction of Jacobi matrices from spectral data, Numer. Math., 44(1984), 317-335. MR0757489 (85i:65052)

[14] P. C. Hansen, Truncated SVD solution to discrete ill-posed problems with ill-determined numerical rank, SIAM J. Sci. Stat. Computing, 11(1991), 503-518. MR1047208 (91e:65058)

[15] A. Horn, On the eigenvalues of a matrix with prescribed singular values, Proc. Amer. J. Math., 76(1954), 4-7. MR0061573(15:847d)

[16] R. A. Horn and C. R. Johnson, Topics in Matrix Analysis, Cambridge University Press, New York, 1991. MR.1091716 (92e:15003)

[17] A. Frieze, R. Kannaa and S. Vempala, Fast Monte-Carlo algorithm for finding low rank approximations, Proceedings of the Foundations of Computer Science, 1998. 378-390, available at http://www.cs.yale.edu/ ${ }^{\sim}$ kannan.

[18] J. Kautsky, N. K. Nichols and P. Van Dooren, Robust pole assignment in linear state feedback, Internat. J. Control, 41(1985), 1129-1155. MR0792933 (86g:93038)

[19] A. W. Marshall and I. Olkin, Inequalities: Theory of Majorization and Its Applications, Academic Press, New York, 1979. MR0552278 (81b:00002)

[20] H. S. Simon and H. Zha, Low rank matrix approximation using the Lanczos bidiagonalization process, SIAM J. Sci. Comput., 21(2000), 2257-2274. MR.1762041(2001e:65065)

[21] F. Y. Sing, Some results on matrices with prescribed diagonal elements and singular values, Canad. Math., 19(1976), 89-92. MR0424850 (54:12808)

[22] J.-G. Sun, Perturbation analysis of the pole assignment problem, SIAM J. Matrix Anal. Appl., 17(1996), 313-331. MR1384510 (97c:93052)

[23] G. W. Stewrat and J.-G. Sun, Matrix Perturbation Theory, Academic Press, Boston, 1990. MR1061154 (92a:65017)

[24] R. C. Thompson, Singular values, diagonal elements, and convexity, SIAM, J. Appl. Math., 32(1977), 39-63. MR0424847 (54:12805)

[25] H. Weyl, Inequalities between two kinds of eigenvalues of a linear transformation, Proc. Nat. Acad. Sci. U.S.A., 35(1949), 408-411. MR0030693(11:37d)

[26] W. W. Wonham, Linear Multivariable Control: A Geometric Control, Third edition. SpringerVerlag, New York, 1985. MR0770574 (86e:93034)

Department of Mathematics, National University of Singapore, 2 Science Drive 2, SINGAPORE 117543

E-mail address: matchudl@math.nus.edu.sg

Department of Mathematics, North Carolina State University, Raleigh, North CarOLINA 27695-8205

E-mail address: chu@math.ncsu.edu 